\title{
Generation and Detection of Multiply-Charged Peptides and Proteins by Matrix-Assisted Laser Desorption Electrospray Ionization (MALDESI) Fourier Transform Ion Cyclotron Resonance Mass Spectrometry
}

\author{
Jason S. Sampson, Adam M. Hawkridge, and David C. Muddiman \\ W. M. Keck FT-ICR Mass Spectrometry Laboratory, Department of Chemistry, North Carolina State \\ University, Raleigh, North Carolina, USA
}

\begin{abstract}
We report the coupling of a hybrid ionization source, matrix-assisted laser desorption electrospray ionization (MALDESI), to a Fourier transform-ion cyclotron resonance mass spectrometer (FT-ICR MS). The details of the source design and initial data are presented. Analysis of peptides and proteins ranging from 1 to $8.6 \mathrm{kDa}$ resulted in high resolving power single-acquisition FT-ICR mass spectra with average charge-states highly correlated to those obtained by nanoESI, thus, providing strong evidence that the ESI process dictates the observed charge-state distribution. Importantly, unlike the recently introduced electrospray assisted laser desorption ionization (ELDI) source reported by Shiea and coworkers $[1,2]$, the data we have obtained to date rely on the use of an organic acid matrix. The results presented herein provide insight into the charging mechanism of this emerging ionization approach, while also expanding the utility of FT-ICR MS for top-down protein and complex mixture analysis. (J Am Soc Mass Spectrom 2006, 17, 1712-1716) (C) 2006 American Society for Mass Spectrometry
\end{abstract}

$\mathrm{T}$ The field of mass spectrometry has grown tremendously over the past century due in large part to the continued development of more powerful ionization techniques, mass analyzer technologies, and ion dissociation techniques. New ionization techniques inherently increase the breadth of applications amenable to mass spectrometry, thereby driving the discovery, development, implementation, and refinement of mass analyzers and ion dissociation methods. The advent of electrospray ionization (ESI) [3] and matrixassisted laser desorption ionization (MALDI) [4, 5] resulted in a paradigm shift in the biological sciences whereby intact biomacromolecules could be ionized and detected by mass spectrometry.

The benefits of direct analysis of samples under ambient conditions with minimal or no sample pretreatment has led to the development of a wide range of novel ionization methods with potentially extraordinary impact in numerous fields (e.g., environmental, forensics, material science, biomedical). These include fused-droplet electrospray ionization (FD-ESI) [6],

Published online September 6, 2006

Address reprint requests to Dr. D. C. Muddiman, W. M. Keck FT-ICR Mass Spectrometry Laboratory, Department of Chemistry, North Carolina State University, Raleigh, NC 27695, USA. E-mail: david_muddiman@ncsu.edu laser desorption atmospheric pressure chemical ionization (LD-APCI) [7], desorption electrospray ionization (DESI) [8], direct analysis in real time (DART) [9], atmospheric-pressure solids analysis probe (ASAP) [10], and electrospray-assisted laser desorption/ionization (ELDI) [1, 2]. Interestingly, modern high-performance mass analyzers are largely based on hybrid technology (e.g., coupling of linear ion trap with a Fourier transform mass spectrometer [11]) and, similarly, so are these new ionization techniques.

Electrospray ionization is inherently well-suited for FT-ICR MS due to the inverse relationship between cyclotron frequency and $m / z$ [12]. Because cyclotron frequency dictates mass resolving power, mass accuracy, limits of detection, and top-down tandem MS efficiencies, the multiple charging (lower $\mathrm{m} / \mathrm{z}$ ) afforded by ESI provides significant advantages [13]. Matrixassisted laser desorption ionization (MALDI) can also be coupled to FT-ICR MS where it can be a very powerful technique for characterizing low molecular weight peptides and metabolites $(<3-5 \mathrm{kDa})$. MALDI is a pulsed ionization technique and ESI is continuous; the ability to analyze multiply-charged clinically-derived low abundant proteins benefits from the ability to interrogate them over long periods of time with multiple MS/MS techniques (e.g., CID, SORI, IRMPD, ECD). 
However, because MALDI generates primarily singly and doubly charged ions, the benefits of FT-ICR MS are not realized for high molecular weight species. Thus, an ionization technique that combines the multiple-charging of ESI and the pulsed nature of MALDI would be highly advantageous for the topdown FT-ICR MS characterization of macromolecules and the analysis of complex mixtures (i.e., high mass resolving power can be obtained without consuming material).

Our specific interests in developing a new ionization source which retained the multiple-charging phenomena but did not require extensive sample preparation and/or on-line separations was for intact [14] and topdown proteomics $[15,16]$ as well as the direct analysis of complex samples (e.g., low molecular weight fraction of serum) $[17,18]$. FT-ICR-MS is uniquely suited for these (and other) applications because these applications demand high mass resolving power and mass accuracy.

Herein, we report the coupling of an ionization source dubbed matrix-assisted laser desorption electrospray ionization (MALDESI) to a Fourier transform ion cyclotron resonance mass spectrometer (FT-ICR-MS). Our ionization source is in principle identical to the recently introduced ELDI source [1,2]. However, unlike ELDI, which does not require matrix, we found that matrix was necessary to obtain appreciable signal. Therefore, to differentiate between pure laser desorption and matrix assisted desorption, we have proposed the acronym MALDESI. Furthermore, our results also support previous observations $[1,2]$ that the detected ions were generated by the electrospray process rather than in the initial matrix-assisted laser desorption event (vide infra). However, if further experimentation reveals that a matrix is not required for a specific application, we fully support the use of the acronym ELDI. The determining factors, which will dictate whether matrix is required (MALDESI) or not (ELDI), will likely be the limit-of-detection and the molecular weight range accessible.

\section{Experimental}

\section{Materials}

Human bradykinin, angiotensin I, and glucagon as well as bovine ubiquitin, 2,5-dihydroxybenzoic acid (DHB), Fluka brand sinapinic acid (SA), and formic acid were purchased from Sigma-Aldrich (St. Louis, MO). HPLC grade acetonitrile and high purity water were purchased from Burdick Jackson (Morristown, NJ). All materials were used as received.

Electrospray solution was prepared by mixing acetonitrile and water $(1: 1 \mathrm{vol} / \mathrm{vol})$ with $0.1 \%$ formic acid. The organic acid matrix was prepared by dissolving either $20 \mathrm{mg}$ of SA or $150 \mathrm{mg}$ DHB in $1 \mathrm{~mL}$ of the electrospray solution. The stainless steel sample target was spotted with a 1:1 ( $\mathrm{vol} / \mathrm{vol})$ mixture of analyte (1 $\mathrm{mg} / \mathrm{mL}$ ) and matrix, covered, and allowed to dry at ambient conditions. ESI experiments containing peptides and proteins were electrosprayed from $1 \mu \mathrm{M}$ solutions.

\section{MALDESI FT-ICR Mass Spectrometer}

Figure 1 shows schematics of the MALDESI source and experimental details. Figure 1a and b show the side and front view, respectively, of our overall experimental configuration of the ionization source. Figure 1c shows an expanded side view of the source. In these experiments, an electrospray solution was directly infused at 400 $\mathrm{nL} / \mathrm{min}$ through a $75 \mu \mathrm{m}$ i.d. fused silica capillary and $30 \mu \mathrm{m}$ tapered PicoTip (New Objective, Woburn, MA) using a Harvard PHD-2000 syringe pump. The MALDESI source was custom built in-house and utilized a $337 \mathrm{~nm}$ pulsed nitrogen laser (Thermo Laser Science, VSL-337ND-S, Franklin, MA). Two UV enhanced aluminum mirrors (part number 10D20AL.2) and a UV fused silica convex lens with a $63 \mathrm{~mm}$ focal length (part number SPX017, Newport Corporation, Irvine, CA) were used to direct and focus the laser beam, respectively. The sample target plate was affixed to a XYZ linear stage (part number 460A, Newport Corporation) for sample positioning.

Figure $1 \mathrm{~d}$ shows the experimental pulse sequence. Mass spectra were acquired in positive-ion mode on a modified ESI-FT-ICR mass spectrometer (IonSpec FTMS Systems, Varian, Inc., Lake Forest, CA) with a 9.4 Tesla superconducting magnet (Cryomagnetics, Oak Ridge, TN). The laser was fired 30 times during a 2-s period, and the ions were accumulated in the external rf-only hexapole [19] while the nanoESI source operated continually. The ions were then ejected from the hexapole, trapped in the ICR cell, excited, and detected. All spectra reported are single-acquisition, collected with $512 \mathrm{k}$ data points with a digitization rate of $1 \mathrm{MHz}$, a Blackman window function applied, and then zerofilled once before fast-Fourier transform.

\section{Control Experiments}

Throughout our investigations, control experiments were always conducted to verify the MALDESI process. These included experiments with the laser being fired without simultaneous ESI to rule out AP-MALDI [20] as the ionization mechanism. Additionally, ESI was established without a laser event to rule out DESI [8] as the ionization mechanism. These data are not shown, but in all cases, the absence of a signal ruled out AP-MALDI or DESI processes as the ionization mechanism. Furthermore, we did not observe signal without the use of an organic acid as a matrix, including analysis of tissue (data not shown), which is contrary to the ELDI reports $[1,2]$ and may reflect specific source design and instrumental parameters. 
(a)
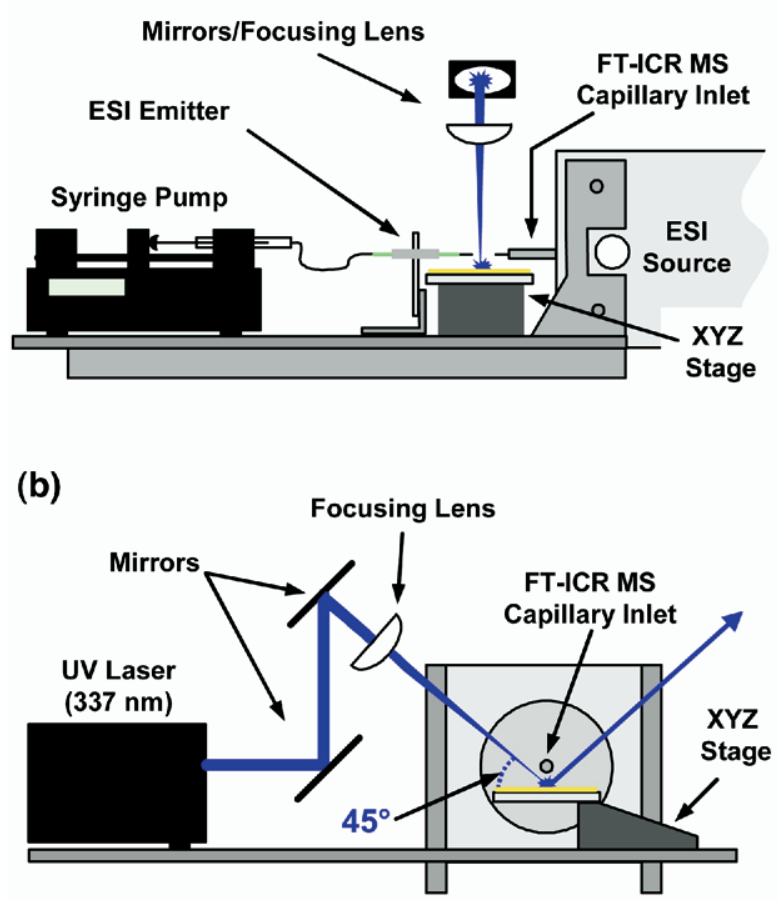

(c)

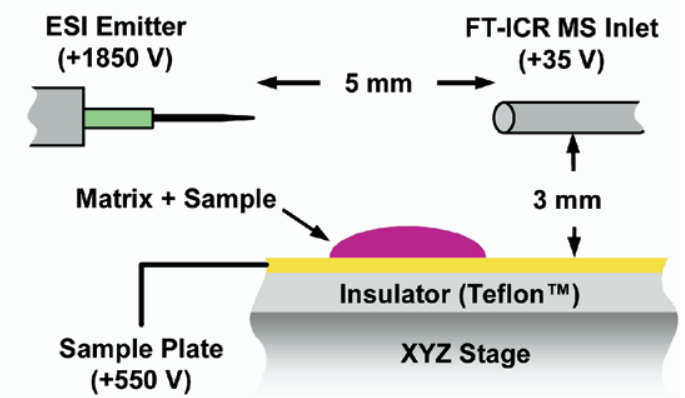

(d)

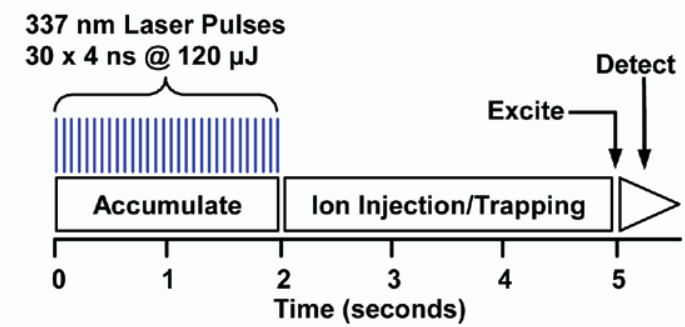

Figure 1. (a), (b), and (c) Front and side views of the MALDESI source. (d) FT-ICR MS experimental pulse sequence.

\section{Results and Discussion}

\section{MALDESI of Peptides and Proteins}

Figure $2 \mathrm{a}$ and $\mathrm{b}$ show the single-acquisition MALDESI (top) and the ESI (bottom) FT-ICR mass spectra of bradykinin and ubiquitin, respectively (see text in figure for details). The neutrals generated from the laser desorption events appear to be captured and charged by the nanoESI droplets. In Figure $2 b$, the inset shows an expansion of the $10+$ charge state of ubiquitin demonstrating isotopic resolution was achieved. It should be noted that the amount of material ablated from the surface is a very conservative estimate and we believe it is significantly less.

Figure $2 \mathrm{c}$ shows the average charge state observed by ESI ( $\mathrm{x}$-axis) and by MALDESI (y-axis) for bradykinin (Figure 2a), angiotensin I (data not shown), glucagon (data not shown), and ubiquitin (Figure 2b). The data is highly correlated (slope $=0.98, \mathrm{R}^{2}=0.9998$ ) providing strong evidence that ionization is occurring via the ESI mechanism.

Figure $2 \mathrm{~d}$ presents approximate neutral capture efficiencies based on the amount of material spotted, size of the spot, laser spot size, number of laser shots, and the absolute abundance of each peptide or protein relative to their corresponding nanoESI spectra. The neutral capture efficiencies range from 0.002 to $0.02 \%$ but we fully expect to be able to improve this with a capture cell or the use of an air amplifier [21-23]. This is an active area of our research where we will utilize natural and stable-isotope labeled peptides to accurately quantify overall capture and ionization efficiencies of the MALDESI process.

\section{Evidence in Support of ESI Charging Mechanism in MALDESI}

To elucidate the role of ESI charging in the MALDESI process, we performed three experiments. First, an equimolar mixture of both angiotensin I and bradykinin were electrosprayed with no laser event (Figure 3a). Second, bradykinin was desorbed solely via the MALDESI process while angiotensin I was produced via conventional ESI (Figure 3b). Third, the angiotensin I was desorbed solely via the MALDESI process while bradykinin was produced via conventional ESI (Figure 3c). Figure 3 a shows the nanoESI FT-ICR mass spectrum of an equimolar mixture of angiotensin I and bradykinin. The $\left[\mathrm{M}+2 \mathrm{H}^{+}\right]^{2+}$ in Figure 3a was observed to be the dominant charge state as in Figure 2c with an abundance ratio of about 4:1 favoring bradykinin. Interestingly, Figure $3 \mathrm{~b}$ shows that even though the bradykinin is produced by the 30 laser shots from the surface and subsequently captured and charged by the ESI droplets, bradykinin remains more abundant than the electrosprayed angiotensin I at $1 \mu \mathrm{M}$. Conversely, in Figure $3 c$ the MALDESI-generated bradykinin remains the dominate species while the electrosprayed angiotensin I is barely observed. This is consistent with the 

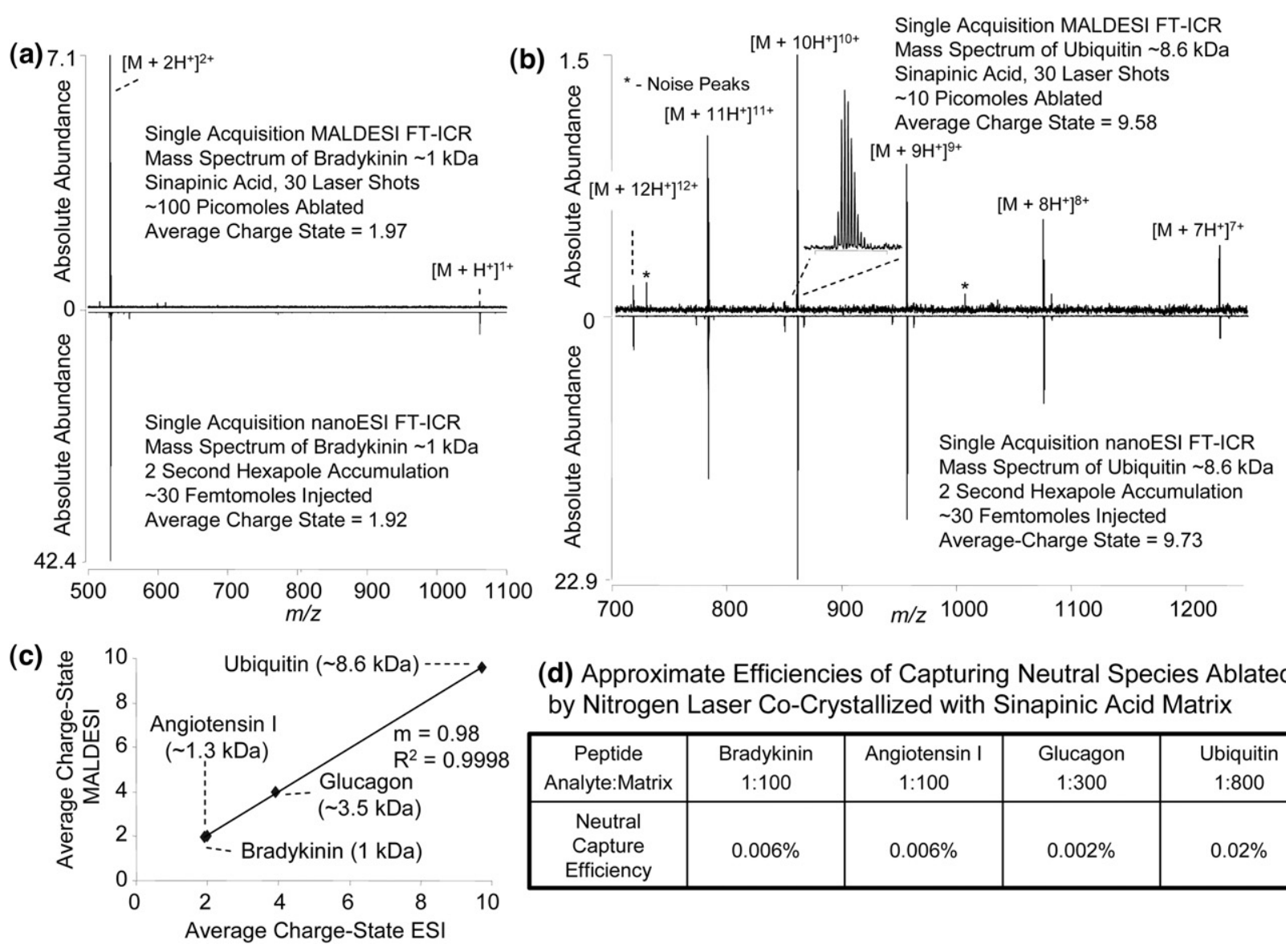
(d) Approximate Efficiencies of Capturing Neutral Species Ablated by Nitrogen Laser Co-Crystallized with Sinapinic Acid Matrix

\begin{tabular}{|c|c|c|c|c|}
\hline $\begin{array}{c}\text { Peptide } \\
\text { Analyte:Matrix }\end{array}$ & $\begin{array}{c}\text { Bradykinin } \\
1: 100\end{array}$ & $\begin{array}{c}\text { Angiotensin I } \\
1: 100\end{array}$ & $\begin{array}{c}\text { Glucagon } \\
1: 300\end{array}$ & $\begin{array}{c}\text { Ubiquitin } \\
1: 800\end{array}$ \\
\hline $\begin{array}{c}\text { Neutral } \\
\text { Capture } \\
\text { Efficiency }\end{array}$ & $0.006 \%$ & $0.006 \%$ & $0.002 \%$ & $0.02 \%$ \\
\hline
\end{tabular}

Figure 2. (a) and (b) MALDESI (top) and nanoESI (bottom with inverted y-axis) FT-ICR mass spectra of bradykinin and ubiquitin, respectively. (c) Plot of the average charge state observed for bradykinin, angiotensin I, glucagon, and ubiquitin via nanoESI (x-axis) and MALDESI (y-axis). (d) Approximate neutral capture efficiencies of the MALDESI source relative to nanoESI of the same peptides.

ESI mechanism observed in Figure 3a where bradykinin out-competes the angiotensin I by a factor of four in the electrospray process. Thus, based on this observation, one would expect angiotensin I to produce a low abundance signal, given the combination of chargecompetition with bradykinin and the lower neutral capture/ionization efficiency of the MALDESI process (Figure 2d).
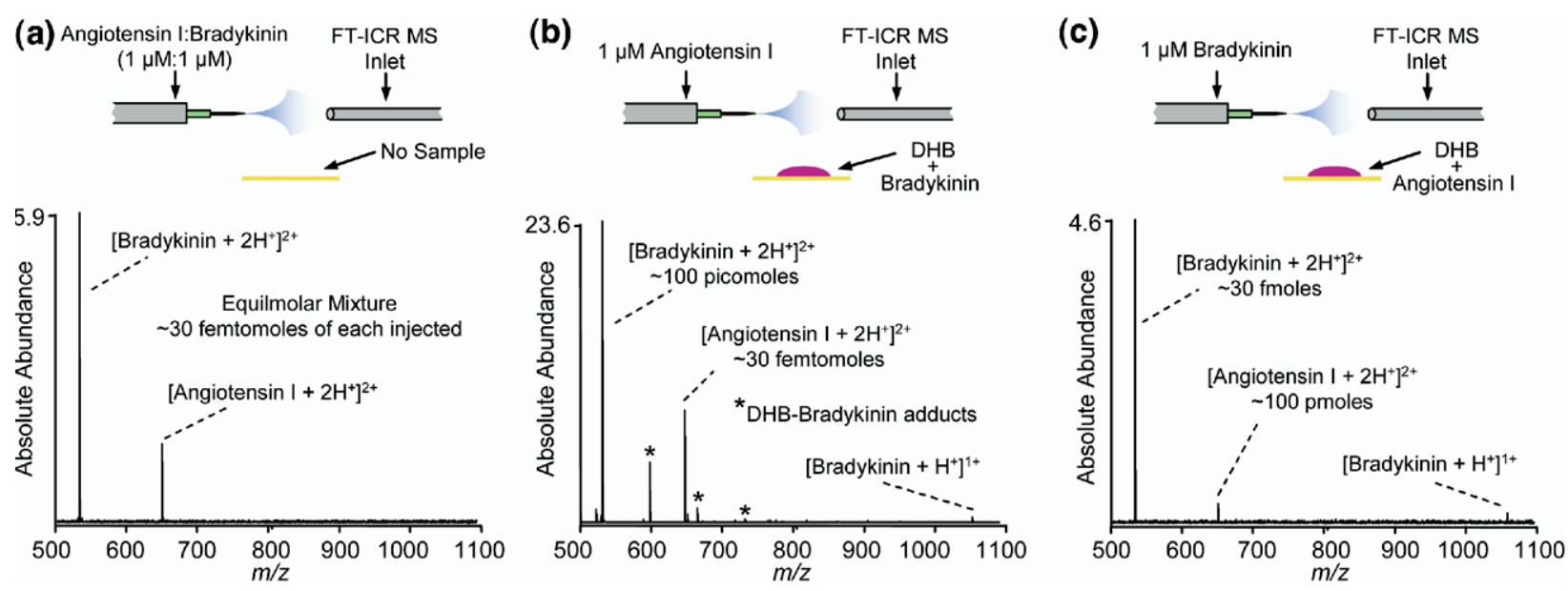

Figure 3. (a) NanoESI FT-ICR mass spectrum of an equimolar mixture of angiotensin I and bradykinin. (b) MALDESI of bradykinin and simultaneous nanoESI of angiotensin I. (c) MALDESI of angiotensin I and simultaneous nanoESI of bradykinin. 


\section{Conclusions}

Clearly, we must better understand the mechanisms of MALDESI before we can confidently apply it to solve real biological problems. Given that MALDESI is a hybrid of MALDI, ESI, and ELDI, there is an enormous amount of experimental space for which to research. We are currently in the process of using stable isotope labeled internal standards to probe the mechanism of MALDESI and to determine the limits of detection of our source. Furthermore, we are actively pursuing the role that the ESI solvent composition, types of organic acid matrix, and matrix crystal morphology play in the generation of MALDESI ions. Finally, we are working on improving sensitivity of the method (e.g., neutral capture efficiency, ion transmission) including the implementation of the air amplifier. These lines of research will result in an optimized and reasonably understood ionization source, which will enable us to commence applications including intact and top-down proteomic analysis using a Q-FTICR as well as direct analysis of biofluids and tissue.

\section{Acknowledgments}

The authors thank Varian, Inc. for the loan of the nitrogen laser used in these studies and the machine shop personnel in the College of Physical and Mathematical Sciences at North Carolina State University for their assistance with the MALDESI source construction. The authors gratefully acknowledge financial support received from the National Cancer Institute, National Institutes of Health (R33 CA105295), the W. M. Keck Foundation, the William R. Kenan, Jr. Fund for Engineering, Technology and Science, and North Carolina State University.

\section{References}

1. Huang, M. Z.; Hsu, H. J.; Lee, J. Y.; Jeng, J.; Shiea, J. Direct Protein Detection from Biological Media through Electrospray-Assisted Laser Desorption Ionization/Mass Spectrometry. J. Proteome Res. 2006, 5(5), 1107-1116.

2. Shiea, J.; Huang, M. Z.; Hsu, H. J.; Lee, C. Y.; Yuan, C. H.; Beech, I.; Sunner, J. Electrospray-Assisted Laser Desorption/Ionization Mass Spectrometry for Direct Ambient Analysis of Solids. Rapid Commun. Mass Spectrom. 2005, 19(24), 3701-3704

3. Fenn, J. B.; Mann, M.; Meng, C. K.; Wong, S. F.; Whitehouse, C. M. Electrospray Ionization for Mass-Spectrometry of Large Biomolecules. Science 1989, 246(4926), 64-71.

4. Karas, M.; Hillenkamp, F. Laser Desorption Ionization of Proteins with Molecular Masses Exceeding 10,000 Daltons. Anal. Chem. 1988, 60(20), 2299-2301.
5. Tanaka, K.; Waki, H.; Ido, Y.; Akita, S.; Yoshida, Y.; Yoshida, T. Protein and Polymer Analysis up to $m / z$ 100,000 by Laser Ionization Time-ofFlight Mass Spectrometry. Rapid Commun. Mass Spectrom. 1988, 2(8), 151-153.

6. Lee, C. C.; Chang, D. Y.; Jeng, J.; Shiea, J. Generating Multiply Charged Protein Ions Via Two-Step Electrospray Ionization Mass Spectrometry. J. Mass Spectrom. 2002, 37(1), 115-117.

7. Coon, J. J.: Harrison, W. W. Laser Desorption-Atmospheric Pressure Chemical Ionization Mass Spectrometry for the Analysis of Peptides from Aqueous Solutions. Anal. Chem. 2002, 74(21), 5600-5605.

8. Takats, Z.; Wiseman, J. M.; Gologan, B.; Cooks, R. G. Mass Spectrometry Sampling Under Ambient Conditions with Desorption Electrospray Ionization. Science 2004, 306(5695), 471-473.

9. Cody, R. B.; Laramee, J. A.; Durst, H. D. Versatile New Ion Source for the Analysis of Materials in Open Air Under Ambient Conditions. Anal. Chem. 2005, 77(8), 2297-2302.

10. McEwen, C. N.; McKay, R. G.; Larsen, B. S. Analysis of Solids, Liquids, and Biological Tissues Using Solids Probe Introduction at Atmospheric Pressure on Commercial LC/MS Instruments. Anal. Chem. 2005, 77(23), 7826-7831.

11. Syka, J. E.; Marto, J. A.; Bai, D. L.; Horning, S.; Senko, M. W.; Schwartz, J. C.; Ueberheide, B.; Garcia, B.; Busby, S.; Muratore, T.; Shabanowitz, J.; Hunt, D. F. Novel Linear Quadrupole Ion Trap/FT Mass Spectrometer: Performance Characterization and Use in the Comparative Analysis of Histone H3 Posttranslational Modifications. J. Proteome Res. 2004, 3(3), 621-626.

12. Henry, K. D.; Williams, E. R.; Wang, B. H.; McLafferty, F. W.; Shabanowitz, J.; Hunt, D. F. Fourier-Transform Mass Spectrometry of Large Molecules by Electrospray Ionization. Proc. Natl. Acad. Sci. U.S.A. 1989, 86(12), 9075-9078.

13. Marshall, A. G.; Guan, S. H. Advantages of High Magnetic Field for Fourier Transform Ion Cyclotron Resonance Mass Spectrometry. Rapid Commun. Mass Spectrom. 1996, 10(14), 1819-1823.

14. Nepomuceno, A. I.; Mason, C. J.; Muddiman, D. C.; Bergen, H. R., III; Zeldenrust, S. R. Detection of Genetic Variants of Transthyretin by Liquid Chromatography-Dual Electrospray Ionization Fourier-Transform IonCyclotron-Resonance Mass Spectrometry. Clin. Chem. 2004, 50(9), 15351543

15. Kelleher, N. L. Top-Down Proteomics. Anal. Chem. 2004, 76(11), 197A203A

16. Reid, G. E.; McLuckey, S. A. “Top Down” Protein Characterization Via Tandem Mass Spectrometry. J. Mass Spectrom. 2002, 37(7), 663-675.

17. Bergen, H. R., III; Vasmatzis, G.; Cliby, W. A.; Johnson, K. L.; Oberg, A. L.; Muddiman, D. C. Discovery of Ovarian Cancer Biomarkers in Serum Using NanoLC Electrospray Ionization TOF and FT-ICR Mass Spectrometry. Dis. Markers 2003, 19(4/5), 239-249.

18. Johnson, K. L.; Mason, C. J.; Muddiman, D. C.; Eckel, J. E. Analysis of the Low Molecular Weight Fraction of Serum by LC-Dual ESI-FT-ICR Mass Spectrometry: Precision of Retention Time, Mass, and Ion Abundance. Anal. Chem. 2004, 76(17), 5097-5103.

19. Senko, M. W.; Hendrickson, C. L.; Emmett, M. R.; Shi, S. D.-H.; Marshall, A. G. External Accumulation of Ions for Enhanced Electrospray Ionization Fourier Transform Ion Cyclotron Resonance Mass Spectrometry. J. Am. Soc. Mass Spectrom. 1997, 8, 970-976.

20. Laiko, V. V.; Baldwin, M. A.; Burlingame, A. L. Atmospheric Pressure Matrix-Assisted Laser Desorption/Ionization Mass Spectrometry. Anal. Chem. 2000, 72(4), 652-657.

21. Hawkridge, A. M.; Zhou, L.; Lee, M. L.; Muddiman, D. C. Analytical Performance of a Venturi Device Integrated into an Electrospray Ionization Fourier Transform Ion Cyclotron Resonance Mass Spectrometer for Analysis of Nucleic Acids. Anal. Chem. 2004, 76(14), 4118-4122.

22. Yang, P.; Cooks, R. G.; Ouyang, Z.; Hawkridge, A. M.; Muddiman, D. C. Gentle Protein Ionization Assisted by High-Velocity Gas Flow. Anal. Chem. 2005, 77(19), 6174-6183.

23. Zhou, L.; Yue, B.; Dearden, D. V.; Lee, E. D.; Rockwood, A. L.; Lee, M. L. Incorporation of a Venturi Device in Electrospray Ionization. Anal. Chem. 2003, 75(21), 5978-5983. 